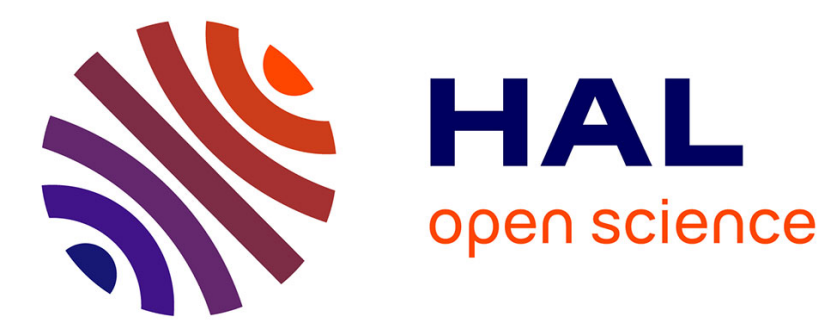

\title{
On computing exact visual hulls of solids bounded by smooth surfaces
}

\author{
Svetlana Lazebnik, Edmond Boyer, Jean Ponce
}

\section{To cite this version:}

Svetlana Lazebnik, Edmond Boyer, Jean Ponce. On computing exact visual hulls of solids bounded by smooth surfaces. IEEE International Conference on Computer Vision and Pattern Recognition (CVPR '01), Dec 2001, Kauai, United States. pp.151-161, 10.1109/CVPR.2001.990469 . inria-00590147

\section{HAL Id: inria-00590147 https://hal.inria.fr/inria-00590147}

Submitted on 3 May 2011

HAL is a multi-disciplinary open access archive for the deposit and dissemination of scientific research documents, whether they are published or not. The documents may come from teaching and research institutions in France or abroad, or from public or private research centers.
L'archive ouverte pluridisciplinaire HAL, est destinée au dépôt et à la diffusion de documents scientifiques de niveau recherche, publiés ou non, émanant des établissements d'enseignement et de recherche français ou étrangers, des laboratoires publics ou privés. 
that is based on two novel representations: the rim mesh, which describes the connectivity of contour generators on the object surface; and the visual hull mesh, which describes the exact structure of the surface of the solid formed by intersecting a finite number of visual cones. We describe the topological features of these meshes and show how they can be identified in the image using epipolar constraints. These constraints are used to derive an image-based practical reconstruction algorithm that works with weakly calibrated cameras. Experiments on synthetic and real data validate the proposed approach.

\section{Introduction}

Most algorithms for surface reconstruction from outlines compute some form of the visual hull [10], or the intersection of solid visual cones formed by back-projecting silhouettes found in the input images. The basic approach dates back to Baumgart's $1974 \mathrm{PhD}$ thesis [1], where a polyhedral visual hull is constructed by intersecting the viewing cones associated with polygonal silhouettes. Volume intersection has remained the dominant paradigm for decades, implemented using representations as diverse as octrees [15] and triangular splines [14]. More recently, graphics researchers have presented efficient algorithms that avoid general 3D intersections by taking advantage of epipolar geometry $[11,13]$. Given an image sequence from a camera undergoing a continuous motion, it is also possible to avoid explicit intersections by reconstructing the visual hull as the envelope of the surface tangent planes along the smoothly deforming occluding contours $[2,3,16]$.

Defined in full generality, the visual hull is the maximal shape consistent with an object's silhouettes as seen from any viewpoint in a given region, and the exact visual hull is the visual hull with respect to a continuous region of space surrounding the object [10]. In this paper we do not treat such limiting cases, but consider the visual hull associated with a finite number of isolated viewpoints (in curves between two viewing cones, and vertices are is points where more than two faces meet. In this con visual hull is exact when it correctly captures the co tivity of these features. Based on this notion of exact hulls, we specify a novel reconstruction algorithm tha not rely on polyhedral intersections or voxel-based ca and produces precise topological and geometric mesh

\section{Preliminaries}

We assume that we are observing a solid object with $\mathrm{x}$ calibrated pinhole cameras. The surface of the obj smooth and without planar patches, and the camer in general position. It is also assumed that apparen tours of the object have been identified in each inpu and oriented counterclockwise, so that the image of th ject always lies to the left of the contour. To simpli presentation, we also assume that contours do not ce singularities such as T-junctions and cusps, and restri attention to objects of genus 0 .

In the rest of the paper, we use the following terr ogy. The rim or contour generator associated with a c is the set of all surface points where the optical ray th the pinhole grazes the object. For general viewpoin rim is a smooth space curve without singularities [4] rims can intersect at isolated points on the surface, frontier points $[3,8,12]$, where the viewing rays fron pinholes lie in the surface tangent plane. The proj of a rim onto the image plane of a camera is the app contour. The set of rays from one camera center p through points on the surface forms the visual cone a ated with that camera. As described in the introductio solid formed by the intersection of all given viewing is the visual hull. Note that the shape of the viewing depends only on the camera center and on the shape object, not on the position of the image plane. Thus jective geometry is sufficient to describe the structure 
converge to create a characteristic X-shape (see Figure 1).

Figure 2 shows an example of an ovoid observed by three cameras. Three viewing cones are drawn, along with intersection curves and rims. The figure shows frontier points and triple points where three viewing cones intersect. In the figure, each frontier point is incident to four rim arcs and four intersection curve branches, and each triple point is incident to six intersection curve branches, only three of which belong to the visual hull. It can be shown that these incidence relations hold in general.

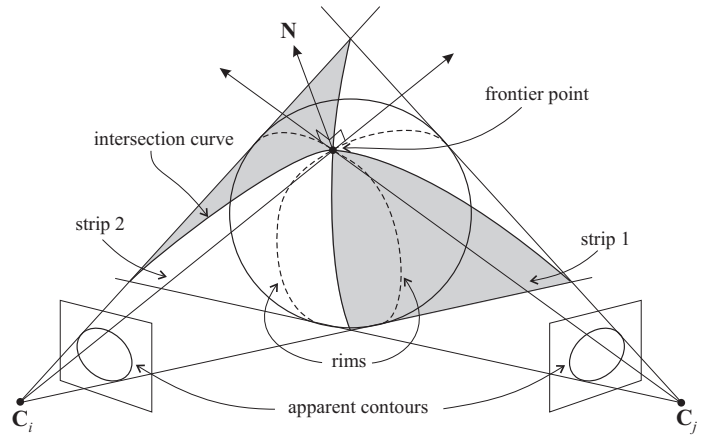

Figure 1: A surface observed by two cameras. The two rims intersect at the frontier point where two cone strips cross.

Now we introduce the two meshes computed by our algorithm. The rim mesh is defined on the surface of the actual object. Its vertices are frontier points, edges are rim segments between two successive frontier points, and faces are regions of the surface bounded by two or more edges. A conceptual precursor of the rim mesh is the epipolar net of Cross and Zisserman [5], who informally discuss, but do not construct, the arrangement of rims on the surface of an object as the camera moves. The visual hull mesh is a topological description of the configuration of visual cone patches on the surface of the solid formed by the intersection of all given visual cones. Its vertices are frontier points (where two strips cross) and triple points (where three cones intersect), edges are intersection curve segments between

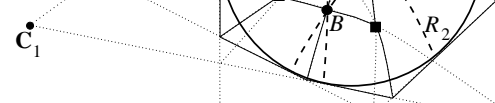

$\dot{\mathbf{C}}_{3}$

$$
\mathbf{C}_{2}^{\bullet}
$$

Figure 2: Configuration of rims and intersection curves ovoid observed by three cameras. Rims are dashed arcs, points are labeled dots, and triple points are squares. Dotte are intersection curve branches outside the visual hull.

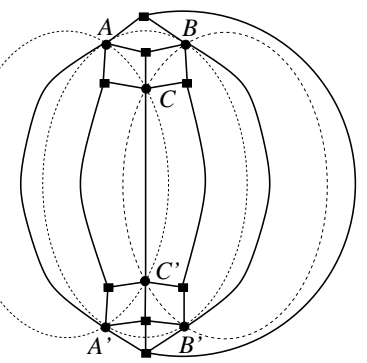

Figure 3: The rim and visual hull meshes of the ovoid from 2. Frontier points are circles, and triple points are squares segments (edges of the rim mesh) are dashed. Intersection segments (edges of the visual hull mesh), are bold lines. No frontier points $A^{\prime}, B^{\prime}$, and $C^{\prime}$ belong to the region of the surfe visible in the previous figure.

\section{Computing the Rim Mesh}

We begin by computing the frontier points, which a vertices of the rim mesh. At a frontier point $\mathbf{P}_{i j}$ views $i$ and $j$, the tangent plane to the surface is al epipolar plane determined by $\mathbf{P}_{i j}$ and the camera $\mathrm{c}$ $\mathbf{C}_{i}$ and $\mathbf{C}_{j}$. In the images, this means that correspo epipolar lines $\mathbf{l}_{i j}$ and $\mathbf{l}_{j i}$ are both tangent to the resp contours at the projections $\mathbf{p}_{i}$ and $\mathbf{p}_{j}$ of $\mathbf{P}_{i j}$. Figur lustrates this basic setup, along with other notation th will need later. Finding a pair of matching frontier po images $i$ and $j$ is a one-dimensional search problem, 
that the corresponding line $\mathbf{l}_{j i}$ in the $j$ th image is tangent to the $j$ th contour. In the presence of contour extraction and calibration errors, there may not exist a pair of matching epipolar lines that exactly satisfy the tangency constraint. In this situation, we find approximately matching frontier points such that the angle difference between the tangent line in one image and the reprojected epipolar tangent from the other image is minimized. Difficulties caused by data error will be further discussed in Section 4.

We obtain all frontier points by matching their projections in two images. The four rim edges incident to a particular frontier point are given by intervals on the two apparent contours that are incident to this point. Thus, there exists a one-to-one correspondence between contour segments in the images and edges of the rim mesh. The orientation of rim edges is then given by the orientation of the corresponding contour segments. In this way, we obtain the complete adjacency information for edges and vertices of the rim mesh. To compute the faces, we need to know the relative ordering in space of the four rim segments incident on each vertex. More formally, we associate with each frontier point $\mathbf{P}_{i j}$ a circular list $I$ where the four edges appear in CCW order around the surface normal. Let $\mathbf{T}_{i}, \mathbf{T}_{j}$ be the tangents to the rims $R_{i}$ and $R_{j}$ at $\mathbf{P}_{i j}$ (see Figure 4). Then the index $i$ appears before the index $j$ in the ordered list $I$ iff

$$
\left(\mathbf{T}_{i} \wedge \mathbf{T}_{j}\right) \cdot \mathbf{N}>0,
$$

where $\mathbf{N}$ is the outward-pointing surface normal (computed as the cross product of the oriented tangent to the contour and the viewing direction).

Equation (1) gives rim ordering in terms of of tangents to the apparent rims, which cannot be computed given only image information. Therefore, we need an equivalent image-based expression for rim ordering. Consider image $i$ and let $\mathbf{v}$ be the direction from the epipole $\mathbf{e}_{i j}$ to $\mathbf{p}_{i}$, the projection of $\mathbf{P}_{i j}$. Let also $\mathbf{t}$ be the tangent to the contour at $\mathbf{p}_{i}$ (see Figure 4), and let $k_{s}$ be the apparent curvature at the surface is elliptic at $\mathbf{P}_{i j}$; or (b) the camera rotate and the surface is hyperbolic. The sign of $\mathbf{v} \cdot \mathbf{t}$ is posi the camera rotates $\mathrm{CCW}$ in the tangent plane and ne otherwise. Moreover, the sign of the apparent curvat is positive if the surface is elliptic or negative if the $s$ is hyperbolic [9]. Thus, expression (2) is positive in th above mentioned cases and negative otherwise. It is fore the desired image-based expression equivalent to

Given the above rim ordering criterion, it is straig ward to trace the loops of edges bounding rim faces. pose we start with one rim segment $s$ of the rim $R_{i}$ anc to find the face that lies to its left. Informally, we tr $s$ along its direction and at its endpoint $\mathbf{P}_{i j}$, simply left turn to get to the next edge, that is, we select the preceding $s$ in the ordered circular list of $\mathbf{P}_{i j}$. We from endpoint to endpoint in this manner, traversing either forward or backward along their orientation, tal left turn each time until we complete a cycle.

\section{Computing the Visual Hull Mes}

As demonstrated in the previous section, we can pute the topology of the rim mesh without knowing thing about its geometry, except for the positions of tier points (note that under weak calibration we car recover these positions up to a projective transforma This topology constrains the adjacency relationship tween triple points and intersection curves, which a vertices and edges of the visual hull mesh.

A triple point $\mathbf{P}_{i j k}$ the intersection of three optica back-projected from contour points $\mathbf{p}_{i}, \mathbf{p}_{j}$, and $\mathbf{p}_{k}$ in different images (see Figure 5). In particular, $\mathbf{p}_{k}$ sa the transfer equation [7]

$$
\mathbf{p}_{k}=\mathbf{l}_{k i} \wedge \mathbf{l}_{k j}=\left(\mathbf{F}_{i k} \mathbf{p}_{i}\right) \wedge\left(\mathbf{F}_{j k} \mathbf{p}_{j}\right),
$$

where $\mathbf{F}_{m n}$ is the fundamental matrix mapping poi image $m$ to epipolar lines in image $n$, and homoge 
$\cdot \mathrm{C}_{i}$

Figure 5: The triple point $\mathbf{P}_{i j k}$ is a "phantom point" where the rays formed by back-projecting epipolar correspondents $\mathbf{p}_{i}, \mathbf{p}_{j}$, and $\mathbf{p}_{k}$ meet in space. $\mathbf{P}_{i j k}$ can be located by tracing the intersection curve $\Gamma_{i j}$ between views $i$ and $j$ and noticing when its projection $\gamma_{i j}$ in the $k$ th image crosses the contour.

coordinates are used for image points. Points $\mathbf{p}_{i}$ and $\mathbf{p}_{j}$ satisfy symmetric equations. Any pair of $\mathbf{p}_{i}, \mathbf{p}_{j}$ and $\mathbf{p}_{k}$ are epipolar correspondents - that is, any two of the points lie in the epipolar plane defined by the two camera centers and one of the points [2]. A triple point is a standard trinocular stereo correspondence, but it does not usually lie on the surface because $\mathbf{p}_{i}, \mathbf{p}_{j}$ and $\mathbf{p}_{k}$ are projections of three different points $\mathbf{P}_{i}, \mathbf{P}_{j}$, and $\mathbf{P}_{k}$ on three different rims.

Just as with finding frontier points, finding triple points is a one-parameter search. We walk along the $i$ th contour in discrete steps and for each contour point $\mathbf{p}_{i}$ find the epipolar line $\mathbf{l}_{j i}=\mathbf{F}_{i j} \mathbf{p}_{i}$ in image $j$, and locate an epipolar correspondent $\mathbf{p}_{j}$ by intersecting $\mathbf{l}_{j i}$ with the $j$ th contour. We then obtain a third point $\mathbf{p}_{k}$ by transferring $\mathbf{p}_{i}$ and $\mathbf{p}_{j}$ using (3) and check whether $\mathbf{p}_{k}$ lies on the $k$ th contour. As shown in Figure 5, transfer of successive epipolar correspondents allows us to trace the intersection curve $\Gamma_{i j}$ between $i$ th and $j$ th cones in the $k$ th image. The triple point is revealed when the traced curve crosses the $k$ th contour.

In general, epipolar correspondents are not unique. In the case shown in Figure 5, each epipolar line intersects each contour twice (this reflects the fact that intersection curves have multiple branches). Moreover, the epipolar correspondence criterion does not say when a triple point belongs to the visual hull - the additional constraint is that the point must not project outside the silhouette in any other input view. However, if we are able to exactly compute the positions of frontier points along the contours, we can use this information to simplify the search for triple points.

Each face of the rim mesh is bounded by rim segments that also belong to the surface of the visual hull, so we can intersection curve, we can identify all the segments b ing a cone strip. Individual faces of the strips are ider by grouping all the intersection curve segments that $\mathrm{p}$ within the contour interval that corresponds to a part rim segment.

The algorithm described above yields the correct hull mesh given exact input data (perfectly extractec tours, error-free fundamental matrices). However, noi calibration error tend to destroy exact topological fea most importantly, intersection curve crossings at fr points. Figure 6 illustrates the situation. The epipolc $\mathbf{1}_{i j}$ is tangent to the contour at point $\mathbf{p}_{i}$ in the $i$ th image line corresponds to the line $\mathbf{l}_{j i}$ in the $j$ th image, which tangent to the $j$ th contour, but intersects it in two ep correspondents $\mathbf{p}_{j 1}$ and $\mathbf{p}_{j 2}$. Intersecting the visua due to these three points in the epipolar plane yield distinct intersection curve points $\mathbf{P}_{i j 1}$ and $\mathbf{P}_{i j 2}$, inst a single frontier point. Thus, instead of being singul intersection curve separates into two distinct branche do not meet. In order to approximate the position of a tier point, we have to match $\mathbf{p}_{i}$ with the epipolar tan point $\mathbf{p}_{j}$ in the $j$ th image. However, the two points lie in the same epipolar plane, and visual rays through do not intersect. We could estimate the location of 1 the midpoint of the segment connecting the points of $c$ approach of the two rays, but this approximated poin not lie on the traced intersection curves. This leads rious consistency problems for a naive implementatio attempts to strictly enforce combinatorial constraints act visual hull structure.

Intuitively, small perturbations to exact contour an ibration data result in contours that back-project to ge cones in space, the intersection of which does not $h$ share the properties of exact visual hulls. For ins while we know that cone strips never break up in (each ray interval along the strip must contain at lea point), in noisy data, they may break up near the fr points as shown in Figure 6. Nevertheless, even with 
rate faces of the rim mesh, and then clip out all components of the curves that project outside any of the silhouettes. In the process, combinatorial information about the curves is maintained, so that it becomes possible to recover the geometry of cone strips. Namely, boundary points of the strips are connected in the order induced by the contour parametrization, and are separated into two groups that correspond to segments bounding the rim on the near and the far side with respect to their distance from the camera. This data structure is a monotone polygon, and it can be triangulated in linear time for purposes of display.

\section{Experimental Results}

The first input sequence consists of six synthetically generated images of an egg model. Contours were extracted using snakes and modeled as cubic B-splines. As seen in Figure 7 , the algorithm correctly generates the rim mesh and the visual hull, both in their topological and geometric form. The contrast between these two forms is clearly visible by comparing two renderings of the same strip in Figure 7 (f) and $(\mathrm{g})$. The exact strip explicitly shows frontier points and triple points, and intersection curves are forced to converge in four branches at frontier points. The triangulated strip does not degenerate at frontier points, because the robust strip tracing algorithm ignores them.

We also demonstrate results for two calibrated nineimage turntable sequences of a gourd and a vase (Figure 8). For both of these data sets, the algorithm constructs a complete rim mesh, even though many of the frontier points are densely clustered near the top and the bottom. To better visualize the structure of these meshes, we rendered their vertices and edges as graphs using a publicly available graph drawing program. The graphs, shown in Figure 8 (b) and (h), reveal the regular structure of rim crossings which is impossible to observe in the images themselves. Each rim mesh in our examples obeys Euler's formula for topological polyhedra of genus $0, V+F=E+2$ (note that the

\section{Discussion and Future Work}

Our preliminary results are intriguing. Significantly, $t$ covery of exact rim meshes has proven to be robus with densely clustered frontier points that do not matching epipolar lines. Note that the rim mesh ture depends only on the relative ordering of frontier along the rims, not on absolute positions - hence the tive stability of the topology. With visual hull meshe situation is different: the connectivity of intersection c and triple points is elusive, while the geometry ma be recovered reliably. It will be important to inves the question of whether these instabilities are inher the conditioning of exact visual hull computation, introduced by our algorithm. We are considering a ent approach to recovering the topology of the visua mesh that would take full advantage of the combin constraints given by the structure of the rim mesh. $V$ also working on extending our implementation to dea T-junctions and surfaces of arbitrary genus, to handle complex and visually interesting objects.

Overall, our approach has several attractive fea Most importantly, it is based on an analysis of the structure of visual hulls from finitely many viewp which has received little attention in previous research approach takes advantage of epipolar geometry and calibration - in a sense, we don't need to know the cameras are. Moreover, our algorithm uses only dimensional computations, and constructs a range of representations, from graph-theoretic and topologic completely image-based, to purely geometric. For reasons, it brings fresh insights to the theory and pr of the venerable problem of visual hull computation.

Acknowledgments. This work was supported in part Beckman Institute, the National Science Foundation grant IRI-990709 and a UIUC-CNRS collaboration ment. 
